COMBÈs Isabelle, ¿Quién mató a Crevaux? Un asesinato en el Pilcomayo en 1882

\title{
Francis Grandhomme
}

\section{OpenEdition}

Journals

Édition électronique

URL : https://journals.openedition.org/jsa/16080

DOI : 10.4000/jsa. 16080

ISSN : 1957-7842

Éditeur

Société des américanistes

Édition imprimée

Date de publication : 15 juin 2018

Pagination : 302-307

ISSN : 0037-9174

Référence électronique

Francis Grandhomme, « combès Isabelle, ¿Quién mató a Crevaux? Un asesinato en el Pilcomayo en 1882 », Journal de la Société des américanistes [En ligne], 104-1 | 2018, mis en ligne le 15 juin 2018, consulté le 03 septembre 2022. URL : http://journals.openedition.org/jsa/16080 ; DOI : https:// doi.org/10.4000/jsa. 16080

Ce document a été généré automatiquement le 3 septembre 2022

Tous droits réservés 


\title{
comBÈs Isabelle, ¿Quién mató a Crevaux? Un asesinato en el Pilcomayo
} en 1882

\author{
Francis Grandhomme
}

\section{RÉFÉRENCE}

COMBÈs Isabelle, ¿Quién mató a Crevaux? Un asesinato en el Pilcomayo en 1882, El País

(Colección Ciencias Sociales e Historia, 41)/CIHA/TEIAA, Santa Cruz de la Sierra, 2017, 225 p., bibliogr., réf. dissém., ill. en noir et blanc, photos en noir et blanc, cartes, tabl.

1 Dans ¿Quién mató a Crevaux?, Isabelle Combès évoque les plages du Río Pilcomayo dans le Chaco bolivien, lieu du massacre en avril 1882 de la mission Crevaux, explorateur français. Une colonie militaire éponyme y est fondée l'année suivante par l'expédition de Daniel Campos, accompagnée par Arthur Thouar, parti à la recherche de son compatriote. L'ouvrage est ainsi consacré à un crime vieux d'il y a 135 ans.

2 Jules Crevaux, médecin de la Marine d'origine lorraine, est alors un héros populaire, rendu célèbre par les récits de ses voyages dans les Guyanes, sur l'Amazone et l'Orénoque publiés dans Le Tour du monde avec le soutien de Jules Ferry. Sa disparition frappe d'autant plus ses contemporains qu'au sommet de sa gloire, il est reparti soudainement en exploration à la fin de 1881 pour établir une liaison entre le Río Paraguay et l'Amazone. Or, dès son arrivée à Buenos Aires, sans doute encouragé par l'explorateur Francisco P. Moreno et le diplomate Santiago Vaca Guzmán, il s'attache à la résolution de l'enclaustramiento bolivien et se lance sur le Río Pilcomayo à la recherche d'une sortie atlantique à la Bolivie, privée de son littoral pacifique par le Chili lors de la guerre du Pacifique (1879-1883). Jusqu'au silence, puis l'annonce en 1882 de son assassinat et de celui de ses compagnons européens et sud-américains, dont son escorte militaire.

On croyait l'affaire classée. La cause est en effet entendue à l'époque : pour les journaux, les savants ou les trois gouvernements - français, boliviens et argentins - qui l'ont 
soutenu, Crevaux, explorateur scientifique désintéressé, est une victime des terribles Indiens toba qui peuplent une partie du Chaco. Mais on comprend au fil de l'ouvrage que toutes les interprétations qu'on donne du massacre sont fausses, ou du moins pourraient l'être.

Isabelle Combès refuse en effet de tenir pour vérité l'histoire officielle et reprend l'affaire à son point de départ. Autant le dire d'emblée, elle ne délivre cependant pas à la fin de son étude le nom de l'assassin. À part la date - et encore -, elle ne peut pas préciser avec certitude l'heure du crime, son lieu, le mobile ou le nombre exact de victimes - et encore moins où se trouvent les cadavres. Il y a là, précise-t-elle, tous les éléments du crime parfait. Et c'est bien sûr là tout l'intérêt du sujet. On se retrouve alors plongé moins à la recherche d'un Percy Fawcett disparu dans le Mato Grosso que dans une véritable intrigue policière digne d'un Sherlock Holmes.

Comme lors de toute réouverture de dossier criminel, Isabelle Combès choisit un nouvel angle d'attaque. Les quelques travaux sur le sujet ont reposé sur des sources françaises, souvent secondaires (Percebois 1980 ; Grandhomme 2011, 2015). Elle, qui est établie à Santa Cruz de la Sierra (Centro de Investigaciones Históricas y Antropológicas, CIHA), mène l'enquête sur place et travaille à partir de sources de première main : en particulier à Sucre, aux Archivo y Biblioteca Nacionales de Bolivia et à la Casa de la Libertad; à Tarija, aux Archivo Histórico, aux Archivo Franciscano et à la collection privée de Virginio Lema Trigo. Des documents originaux ou oubliés, dont plusieurs sont reproduits en annexes, sont exhumés, telle une série de dessins de Théophile Novis, compagnon de Thouar lors de sa troisième expédition (voir aussi Novis 2017) ou des témoignages de Natalio Roldán ou de Leocadio Trigo. Cette capacité à mobiliser des sources originales est à saluer parce que, pour certaines d'entre elles, on ne connaissait que des copies qui apparaissent dès lors truffées d'erreurs ou d'approximations. Reprenant les témoignages, ou en convoquant de nouveaux, elle relève les contradictions de dates, de lieux, d'identités, et tisse une nouvelle présentation des événements : les scénarios évoluent, des accusateurs deviennent des accusés, des témoins des complices.

En effet, dans un premier chapitre, Isabelle Combès évoque d'abord ce que l'on appelle "L'affaire Crevaux ». Avec sa scène de crime, l'axe du Pilcomayo. Avec ses suspects, les groupes indigènes, une véritable mosaïque, dont émergent les Toba dans un Chaco alors dernier bastion de résistance indienne à la colonisation; mais aussi des missionnaires franciscains dont le chef, l'Italien Doroteo Giannecchini, aide Crevaux dans ses préparatifs, mais qui est jaloux de sa mainmise spirituelle et économique sur les Indiens et craint que la sécularisation accompagne la colonisation; et des colons miséreux et métissés du bourg de Caiza qui, à la suite d'un vol de chevaux, viennent de mener une expédition punitive sanglante contre les Indiens, sans se soucier que leur vengeance pourrait alors s'exercer contre Crevaux. L'affaire apparaît aussi comme la chronique d'une mort annoncée où une émissaire toba, la jeune Yalla-Petrona, fille du cacique Caligagae, joue un rôle trouble et où chacun des protagonistes exprime des mauvais pressentiments ou formule des mises en garde avant le départ. Jusqu'à l'annonce de la mort de Crevaux rapportée par l'Indien Yahuanahua et publiée à la une des journaux.

7 Intitulé " À la recherche des restes de la mission Crevaux », le deuxième chapitre traite de l'enquête. Plusieurs expéditions partent à la recherche du lieu du crime : Fontana, puis Rivas et Giannecchini en 1882 ; Ibaceta, puis Campos et Thouar l'année suivante. Tels des enquêteurs, certains se mettent à l'œuvre, relayés par la presse (El Trabajo ou La Estrella de Tarija) et relèvent les premiers indices, les premières confidences : d'Indiens, d'un ancien 
captif des Toba, José Correa, de Yalla-Petrona et même d'un jeune rescapé, le Bolivien Francisco Zeballos. Des armes et des effets personnels des explorateurs sont retrouvés. Un prétendu crâne de Crevaux est même exhibé par Thouar ainsi que, véritable trophée, celui de son assassin présumé Cuserai. On fait fausse route : après cinq ans d'espoirs, tout n'apparaît que dissimulations, mensonges et impostures. Yahuanahua est-il impliqué dans le massacre? ou Iramaye, l'interprète de l'expédition? Que croire des multiples versions données par Correa ou par Thouar "l'imposteur français », avide de gloire? Quant à Giannecchini n'est-il que la victime des erreurs de Thouar qui l'accuserait de complicité avec les Indiens pour se dédouaner?

Les « Questions en suspens » sont l'objet du troisième chapitre, entre bruits et rumeurs. La date reste le sujet le moins discutable, les $24,25,26$ ou plutôt 27 avril 1882, que retient Isabelle Combès. Le nombre de victimes, entre 17 et 35 selon les sources, mais quelle est leur identité ? Cinq Français, mais pas Joseph Didelot, le secrétaire de Crevaux, resté à Buenos Aires. Quatre soldats boliviens et argentins, mais qui sont-ils vraiment? et combien étaient les accompagnateurs locaux? Le lieu du crime, Teyu, capitale des terribles Toba, mais le toponyme n'est pas pour les Indiens attaché à un lieu précis. Sur le mobile des tueurs et le modus operandi, en particulier l'arme du crime. La préméditation doit-elle être retenue? Et quelle a été l'attitude des explorateurs? Ne se sont-ils pas défendus? Les incertitudes libèrent l'imagination et donnent corps à des légendes et même à une littérature tragicomique où l'on parle du cannibalisme supposé des Toba, de deux survivants atrocement suppliciés ou d'un autre imaginaire. Enfin, l'histoire s'estelle répétée avec la disparition en 1899 de l'Espagnol Enrique de Ibarreta?

9 L'identité des coupables reste tout aussi incertaine. Ces « Assassins sans visage », titre du dernier et quatrième chapitre, prennent d'abord une figure indienne. Mais dans ce kaléidoscope ethnique aux lieux de réunion communs est-ce celle des Toba ou celle des Noctenes (actuels Weenhayek) comme le prétendent les premiers, ou de tout autre groupe, chacun ciblant les ennemis de toujours? Désigne-t-on d'ailleurs les auteurs ou leurs chefs? De plus qui se cacherait derrière? Les instigateurs auraient-ils un visage pâle ? Celui des Pères? Mais Thouar, qui les accuse à partir de 1887, vitupère plus qu'il ne fournit de preuves. D'ailleurs ceux-ci savent très bien qui est Crevaux et auraient tout à craindre d'être mêlés à sa mort. Cependant, les accusations qu'ils retournent contre les créoles sont tout aussi génériques et parmi les victimes de la mission Crevaux nombreux sont de leur parenté. Isabelle Combès conclut alors que Crevaux est mort pour ce qu'il représente : voyageur scientifique au service de la civilisation, il incarne pourtant aux yeux des Indiens la colonisation, dont il est bien un agent même s'il en critique les effets collatéraux (comme Cuserai est accusé car il incarne la résistance des Toba), ce qui irrite les colons. Le détonateur du massacre paraît cependant l'expédition des Caiceños et la logique du cycle de représailles Indiens/colons. À qui profite alors le crime? La mort de Crevaux, érigé en martyr de la civilisation, est le prétexte idéal à une colonisation par les États, mais au profit surtout de l'Argentine, dans une volonté d'intégration territoriale (l'enjeu est pourtant majeur pour la Bolivie face au Paraguay, l'affaire Crevaux peut ainsi être évoquée comme un élément de la pré-guerre du Chaco, qui inscrit l'antériorité de la présence bolivienne).

¿Quién mató a Crevaux? nous offre donc une enquête des plus intéressantes. Mais pourquoi un livre entier? En effet, le propos parfois très factuel paraît se répéter avec un sentiment de tourner autour du sujet. Le style est pourtant de grande qualité, le plan clair, les titres courts et évocateurs. Le souci du détail n'alourdit pas la thèse, il la 
soutient. Car, dans une affaire où tous les protagonistes sont suspects, démêler les choses est essentiel. Des erreurs ou approximations qu'on considérait jusqu'à présent comme fortuites apparaissent ainsi volontaires, propres à entretenir le flou. Comment en effet établir le déroulement des faits si tout - date, lieu, acteurs, mobile - est sujet à caution? Isabelle Combès a donc cherché la preuve. Les conclusions même partielles qu'elle apporte constituent désormais une réponse définitive à certaines questions posées. Même si l'énigme n'est globalement pas résolue, certains ressorts de l'affaire Crevaux sont établis une fois pour toutes. Son travail est aussi une illustration des mécanismes et des ressorts des bruits et rumeurs. Elle apporte réellement un regard neuf, très documenté et fouillé, plus global et complet que d'autres publications récentes qui évoquent l'affaire Crevaux (Fenchelle-Charlot 2014 ; Jung 2017).

11 Le livre est aussi à replacer dans une œuvre. Dans la suite de ses précédentes publications, telle la Historia del pérfido Cuñamboy (2016), elle s'attache en effet à récrire l'histoire dans une optique intégrant les indigènes (qui, eux, n'ont pas produit de défense écrite à l'époque de l'affaire Crevaux). Isabelle Combès démonte ainsi l'argumentaire qui, par principe, rend les Toba responsables, en démontrant que la confusion rend difficile pas tant l'identification des auteurs matériels du crime que celle de leurs inspirateurs. En effet, en bonne anthropologue, elle porte un regard que n'avaient guère les analyses antérieures (ce qui les rend donc en partie inopérantes). Et si elle parle d'" assassins sans visage ", elle en donne un à ces Indiens, dont on comprend qu'ils ne peuvent être réduits à des «tigres " pour reprendre le terme de 1882 d'Eudogio Raña, sous-préfet du Chaco bolivien (même si certains clichés restent tenaces comme le prétendu cannibalisme des Toba dont Crevaux aurait été victime). Isabelle Combès estime ainsi que le massacre de la mission Crevaux est plus une querelle entre Blancs - missionnaires et colons - qu'entre Blancs et Indiens, dont l'enjeu est moins l'ouverture d'une voie de communication par le Río Pilcomayo que l'appropriation du Chaco par la conquête et la colonisation. L'ouvrage analyse donc aussi les ressorts locaux de la conquête territoriale des États sudaméricains en concurrence.

12 Aussi le titre de l'ouvrage s'éclaire. Car, si Isabelle Combès bien qu'anthropologue ne hait pas tous les explorateurs (mais seulement Thouar l'archétype du conquérant ?), ce n'est pas le sort de Crevaux qui est fondamentalement au cœur de l'ouvrage. Elle s'intéresse plus au lieu Crevaux qu'à la personne de Crevaux. Elle éprouve certes de l'empathie à son égard, mais au passage elle ne l'épargne pas. Pour ses certitudes d'Européen de la fin du XIX ${ }^{e}$ siècle, un agent colonisateur accompagné par des soldats boliviens et argentins, uniformes dont la présence a sans doute scellé le sort. Elle écorne ainsi le «mythe Crevaux », y compris ses compétences: la recherche fait "peu de cas que le Français a conduit à la mort une quinzaine de personnes » remarque-t-elle avec justesse et, par ailleurs, indépendamment du contexte local troublé, rien ne dit qu'il aurait réussi.

Le livre n'est ainsi pas tant la recherche de la résolution de l'énigme que la compréhension du mécanisme d'interprétation du crime en son temps. Donner le nom de l'assassin importe peut-être moins que d'établir comment s'est construite la version officielle de la culpabilité indienne, les autres hypothèses ayant été éludées pour des raisons partisanes (même si le mouvement de sécularisation explique aussi les attaques contre les "perfides missionnaires»). Il s'agit de renvoyer en Europe l'image de «sauvages indigènes» que seule la civilisation et donc la colonisation pourraient maîtriser. En 1882, quelques mois après la disparition non moins médiatisée de la mission Flatters dans le Sahara, les tenants de l'expansion mondiale, en particulier Jules Ferry, 
peinent à populariser leur politique. La figure héroïque de Crevaux alimente ainsi ses propagandistes, notamment par le relais du Tour du monde ou d'autres périodiques comme le Journal des voyages et ceux des sociétés de géographie. L'analyse faite à l'époque renvoie moins à des faits établis qu'à l'imaginaire européen.

Le mystère de la disparition de l'explorateur français Jules Crevaux demeure, certes. Grâce au travail d'Isabelle Combès, il ne reste cependant pas entier et la vision que l'on a désormais est plus proche de la vérité, quitte à écorner le « mythe Crevaux ».

\title{
BIBLIOGRAPHIE
}

\author{
COMBÈs Isabelle
}

2016 Historia del pérfido Cuñamboy. La Cordillera chiriguana en los albores de la independencia de Bolivia, Itinerarios Editorial (Scripta autochtona, 16)/ILAMIS, Cochabamba.

FENCHELLE-CHARLOT Corinne

2014 Jules Crevaux. L'explorateur de l'Amazonie, Gérard Louis éditeur, Haroué.

\section{GRANDHOMME Francis}

2011 Une figure lorraine : Jules Crevaux (1847-1882) et l'exploration de l'Amérique du Sud, thèse de doctorat en histoire, université Nancy 2.

2015 «"Partir, réussir ou mourir ?" Rumeurs autour du voyage sans retour d'un explorateur de l'Amérique du Sud au XIX siècle », Annales de l'Est, numéro spécial, p. 125-148.

JUNG Didier

2017 Émile Arthur Thouar. De l'île de Ré à la jungle bolivienne, Le Croît Vif, Saintes.

\section{NOVIS Teófilo}

2017 El Chaco en Imágenes (1887). Introducción y edición de Isabelle Combès, Publicaciones Casa de la Libertad, Sucre.

PERCEBOIS Gilbert

1980 « Les explorations et la mort tragique de Jules Crevaux vues par ses contemporains nancéiens ", Actes du 104 Congrès national des Sociétés savantes, p. 69-79.

\section{AUTEURS}

\section{FRANCIS GRANDHOMME}

Centre régional universitaire lorrain d'Histoire (CRULH), Nancy 